\title{
Technical note: An evaluation of technology-recorded rumination and feeding behaviors in dairy heifers
}

\author{
M. A. Reynolds, ${ }^{1,2,3}$ M. R. Borchers, ${ }^{1 *}$ J. A. Davidson, ${ }^{3}$ C. M. Bradley, ${ }^{3}$ and J. M. Bewley ${ }^{1,4}$ \\ ${ }^{1}$ Department of Animal and Food Sciences, University of Kentucky, Lexington 40546 \\ ${ }^{2}$ Department of Animal Science, University of Nebraska-Lincoln, Lincoln 68527 \\ ${ }^{3}$ Purina Animal Nutrition Center, Gray Summit, MO 63039 \\ ${ }^{4}$ Alltech Inc., Nicholasville, KY 40356
}

\section{ABSTRACT}

Precision dairy monitoring technologies have become increasingly popular for recording rumination and feeding behaviors in dairy cattle. The objective of this study was to validate the rumination and feeding time functions of the CowManager SensOor (Agis, Harmelen, the Netherlands) against visual observation in dairy heifers. The study took place over a $44-d$ period beginning June 1, 2016. Holstein heifers equipped with CowManager SensOor tags attached according to manufacturer specifications $(\mathrm{n}=49)$ were split into 2 groups based on age, diet, and housing type. Group 1 heifers $(\mathrm{n}=24)$ were calves (mean $\pm \mathrm{SD}) 2.0 \pm 2.7$ mo in age, fed hay and calf starter, and housed on a straw-bedded pack. Group 2 heifers $(\mathrm{n}=25)$ were $17.0 \pm 1.3 \mathrm{mo}$ in age, fed a TMR, confirmed pregnant, and housed in freestalls. Visual observation shifts occurred at 1500, 1700, 1900, and 2100 h. Each heifer was observed for 2 hour-long periods, with both observation periods occurring on the same day. Visual observations were collected using a synchronized watch, and "start" and "stop" times were recorded for each rumination and feeding event. For correlations, data from CowManager SensOor tags and observations were averaged, so a single 1-h observation was provided per animal, reducing the potential for confounding repeated measures being collected for each animal. Concordance correlations (CCC; epiR package; R Foundation for Statistical Computing, Vienna, Austria) and Pearson correlations (r; CORR procedure; SAS Institute Inc., Cary, NC) were used to calculate association between visual observations and technology-recorded behaviors. Visually observed rumination time was correlated with the CowManager SensOor $(\mathrm{r}=0.63, \mathrm{CCC}=0.55)$.

Received September 1, 2018.

Accepted March 19, 2019.

*Corresponding author: matthew.borchers@uky.edu
Visually observed feeding time was also correlated with the CowManager SensOor $(r=0.88, C C C=0.72)$. The difference between technology-recorded data and visual observation was treated as the dependent variable in a mixed linear model (MIXED procedure of SAS). Time of day, age in months, and group were treated as fixed effects. Individual heifers were treated as random and repeated effects. The effects of time of day, age, and group on rumination and feeding times were not significant. The CowManager SensOor was more effective at recording feeding behavior than rumination behavior in dairy heifers. The CowManager SensOor can be used to provide relatively accurate measures of feeding time in heifers, but its rumination time function should be used with caution.

Key words: rumination behavior, feeding behavior, precision dairy monitoring

\section{Technical Note}

Variation in activity, rumination, and feeding behavior can be used to identify cows with health concerns (Schirmann et al., 2016). Dry matter intake has been shown to decrease with the onset of ruminal acidosis (Owens et al., 1998), displaced abomasum (Van Winden et al., 2003), and subclinical ketosis (Duffield, 2000; Goldhawk et al., 2009). Reductions in rumination activity have been linked to metabolic disorders (Hansen et al., 2003; DeVries et al., 2009), mastitis (Fogsgaard et al., 2012), anxiety (Bristow and Holmes, 2007), and stress from regrouping (Schirmann et al., 2011). Additionally, Edwards and Tozer (2004) showed that cows diagnosed with ketosis, left-displaced abomasum, and digestive disorders could be detected before clinical diagnosis using technology-recorded changes in walking activity.

The CowManager SensOor (Agis, Harmelen, the Netherlands) was developed primarily as an earattached estrus-detection aid for mature cattle that also provides health information. Rumination, feeding 
behavior, and activity are recorded through ear movement via a 3-dimesional accelerometer located in the ear tag. The CowManager SensOor is positioned on an electronic identification tag placed in the middle of the cow's left ear. It is approximately $28 \mathrm{~g}$, resembles an ordinary ear tag, and measures $5.1 \mathrm{~cm}$ long, $6.4 \mathrm{~cm}$ wide, and $1.9 \mathrm{~cm}$ thick at its largest points. The CowManager SensOor battery is guaranteed for 5 years. Data from the sensor are collected through a router and sent to the producer's computer, where the data are stored. Data for individual animals are continuously collected each minute and are classified in 1 of 5 ways: high active, active, not active, eating, or ruminating. The high-active classification is used to identify estrus behavior, and the not-active classification identifies lack of activity. However, these metrics were not used in the current study, as this experiment focused solely on the rumination and eating functions of the sensor.

Previous evaluations of the CowManager SensOor have been conducted with lactating dairy cattle (Bikker et al., 2014; Borchers et al., 2016) but to the authors' knowledge, an evaluation of the technology has not been conducted using dairy heifers. The purpose of this experiment was to compare technology-recorded rumination and feeding behavior in dairy heifers with visually observed rumination and feeding behavior. We hypothesized that visually observed and CowManagerrecorded rumination and feeding behaviors for heifers would be highly correlated.

The study took place at the Heifer Innovation Unit (Purina Animal Nutrition Center, Gray Summit, MO) over a 44-d period beginning June 1, 2016. Sample size was determined using the methods of Friedman (1982). All power tests were calculated to obtain a power (1 $-\beta)$ of 0.90 and a type-I error probability $(\alpha)$ of 0.05 (2-tailed). An effect size equivalent to correlation coefficients was used (rumination time $\mathrm{r}=0.93$, feeding time $\mathrm{r}=0.88$; Bikker et al., 2014). A minimum of 24 heifers were needed to meet power requirements, and this number was increased to 49 heifers to account for potential instances of missing data. Holstein heifers equipped with CowManager SensOor tags (CowManager version 7.0), attached according to manufacturer specifications $(\mathrm{n}=49)$ were split into 2 groups based on age, housing, and diet type.

Group 1 heifers $(\mathrm{n}=24)$ were weaned calves (mean $\pm \mathrm{SD}$ ) $2.0 \pm 2.7 \mathrm{mo}$ in age. Group 1 heifers were fed chopped alfalfa hay at $1100 \mathrm{~h}$ and calf starter grain (AmpliCalf Grower, Purina Animal Nutrition LLC, Shoreview, MN) at $1200 \mathrm{~h}$. Group 1 heifers were housed on a straw-bedded pack throughout the study. Group 2 heifers $(\mathrm{n}=25)$ were $17 \pm 1.3$ mo in age. Group 2 heifers were confirmed pregnant and housed in freestalls bedded with composted mulch. Group 2 was provided
TMR containing lactating cow refusals, grass hay, and a supplemental complete feed (HeiferSmart 22 Complete Feed, Purina Animal Nutrition LLC) at $1400 \mathrm{~h}$. Fly-avoidance behaviors were not recorded during the study, but very few flies were noted in the barn.

Observations were collected from vantage points at the periphery of each pen. The observer changed vantage points to constantly maintain visual contact with animal subjects. Visual observation shift times were determined after the most common eating times were observed before the study. The most common eating time was determined to be after feed delivery for both groups, at 1500, 1700, 1900, and 2100 h. Each heifer was observed for two 1-h periods, with both observation periods occurring on the same day. Observation shifts lasted $1 \mathrm{~h}$ and $10 \mathrm{~min}$. Heifers were observed for $5 \mathrm{~min}$ before and after the hour of observation, to decrease the chance of missing an activity before or after the observation shift. Observed heifers were selected from a randomized list of all heifers on the day of observation. Previously selected animals were removed from consideration to avoid duplication of observations. Heifers displaying signs of estrus were excluded for that day and reassigned to another day, to remove any potential effects on recorded rumination and feeding behaviors, and replaced with another heifer. The duration of each behavioral event was recorded in hours, minutes, and seconds. "Start" and "stop" times were recorded for each rumination and eating activity, using a satellite-synced watch (Casio America Inc., Dover, NJ). These times were then used to calculate the duration of each behavior during the observation shift. Similar to Schirmann et al. (2009), we defined the onset of rumination as the regurgitation of a new bolus. The observer recorded the "start" when the bolus reached the mouth, after traveling up the esophagus, and chewing of the bolus ensued. The "stop" time was recorded when the bolus was swallowed. Subsequent rumination bouts were treated as separate events. Eating behavior was observed similar to Borchers et al. (2016), who classified a cow as eating if she were standing at the feed bunk and actively ingesting or chewing feed. If chewing stopped for longer than $5 \mathrm{~s}$, the eating event was recorded as stopped. Time spent chewing away from the feeding area was not considered feeding behavior.

Observations for each heifer were averaged to provide a single estimate for each heifer, removing potential confounding effects of repeated measures in the correlations. Concordance correlation coefficients (CCC; epiR package; R Foundation for Statistical Computing, Vienna, Austria) and Pearson correlation coefficients (r; CORR procedure of SAS version 9.3; SAS Institute Inc., Cary, NC) evaluated association between visual observations and technology-recorded behaviors. Con- 
cordance correlation coefficients represent concurrent measures of correlation as well as accuracy and precision (Lin, 1989; Bikker et al., 2014). Differences in standard deviation between 2 measures are indicated by the scale shift $(\mu)$, and over- or underprediction is represented by location shift ( $V$; Lin, 1989; Bikker et al., 2014). Scale and location shifts are used to calculate the bias correction factor $\left(C_{b}\right)$ and are multiplied by the Pearson correlation to make the CCC. Mixed linear models (MIXED procedure of SAS) were used to evaluate potential reasons for differences between visual observations and technology data. Differences between observed behaviors and technology-recorded data were treated as dependent variables, and time of day, age in months, and group were treated as fixed effects. All 2 -way interactions were considered in the model. Nonsignificant $(P \geq 0.05)$ interactions were removed using backward-stepwise elimination. Each heifer was treated as a random intercept. Repeated measurements of individual heifers were addressed using a compound symmetry covariance structure to account for correlation of errors within subjects, with the correlation considered constant across each time.

Minimum and maximum air temperatures were recorded over the course of the study (National Weather Service, National Oceanic and Atmospheric Administration, St. Louis, MO). Mean minimum temperature was $21.8 \pm 2.9^{\circ} \mathrm{C}$, and mean maximum temperature was $32.1 \pm 3.9^{\circ} \mathrm{C}$. CowManager rumination time was directly compared with visual observation over a $1-\mathrm{h}$ period. The correlations between visually observed rumination and the CowManager SensOor are shown in Table 1. Results were similar to those of Borchers et al. (2016), showing moderate correlation between visual observation and technology-recorded rumination $(\mathrm{r}=$ $0.69, P<0.01 ; \mathrm{CCC}=0.59)$. However, our results contradict those of Bikker et al. (2014), who found strong correlation between visual observation and technologyrecorded rumination $(\mathrm{r}=0.93, P<0.01 ; \mathrm{CCC}=0.93)$. Borchers et al. (2016) and Bikker et al. (2014) included lactating dairy cattle in experiments, but the present experiment recorded activity on heifers.

Table 1. Measures of correlation, accuracy, and precision ${ }^{1}$ between technology-recorded $^{2}$ rumination and feeding behavior and visual behavior observation

\begin{tabular}{lccccc}
\hline Behavior & $\mathrm{r}$ & $\mathrm{C}_{\mathrm{b}}$ & $\mathrm{CCC}$ & $\mathrm{V}$ & $\mu$ \\
\hline Rumination & 0.63 & 0.88 & 0.55 & -0.31 & 1.51 \\
Feeding & 0.88 & 0.83 & 0.72 & -0.64 & 1.02 \\
\hline
\end{tabular}

${ }^{1}$ Pearson correlation coefficients $(\mathrm{r})$, bias correction factor $\left(\mathrm{C}_{\mathrm{b}}\right)$, concordance correlation coefficients (CCC), location shift (V), and scale shift $(\mu)$.

${ }^{2}$ Cow Manager SensOor ear tag (Agis, Harmelen, the Netherlands).
The CowManager-recorded feeding time was directly compared with visual observation over a 1-h period. The visually observed feeding times were highly correlated with the sensor and are shown in Table 1. Results were similar to those of Bikker et al. $(2014 ; \mathrm{r}=0.88$, $P<0.01 ; \mathrm{CCC}=0.75)$ and Borchers et al. $(2016 ; \mathrm{r}$ $=0.88, P<0.01 ; \mathrm{CCC}=0.82)$. In the current study, heifers were fed different diets depending on group. Group 1 heifers were fed alfalfa hay and grain. Similar to Borchers et al. (2016), Group 2 heifers were fed a TMR. The cattle from Bikker et al. (2014) were fed different diets due to another ongoing experiment: 11 cows were fed a partial mixed ration, and 4 cows were fed a TMR. In the current study, both groups of heifers were fed once daily. Bikker et al. (2014) also fed cattle once daily, through roughage intake control feeders at 1000 h. Borchers et al. (2016) fed cattle twice daily at $0530 \mathrm{~h}$ and $1330 \mathrm{~h}$ in a raised feed bunk. Regardless of the investigated diets or the methods of feeding used, the CowManager technology was consistent when measuring feeding behavior in heifers.

Fixed effects of age, group, and time of day on rumination and feeding behavior are shown in Table 2. No significant 2-way interactions were found between fixed effects in any model. The lack of significance in age or group indicates that the CowManager system could be used to record rumination and feeding behaviors in heifers ranging in age from 2 to 17 mo and across housing types (straw-bedded pack or freestalls). The lack of significance of the time of day on feeding behavior indicates that the CowManager technology was consistent in recording rumination and feeding behaviors throughout the study. The tendency $(P=0.06)$ for time of day to influence rumination may be caused by feed delivery times and the timing of rumination following feeding.

Table 2. Type 3 tests of fixed effects from a mixed linear model comparing technology-recorded ${ }^{1}$ rumination and feeding behavior with visually observed behaviors in dairy heifers

\begin{tabular}{lcccc}
\hline $\begin{array}{l}\text { Behavior and } \\
\text { variable }\end{array}$ & $\begin{array}{c}\text { Numerator } \\
\text { df }\end{array}$ & $\begin{array}{c}\text { Denominator } \\
\text { df }\end{array}$ & $F$-value & $P$-value \\
\hline Feeding & 1 & 45 & 0.19 & 0.67 \\
$\quad$ Age $^{2}$ & 1 & 45 & 1.35 & 0.25 \\
Group $^{3}$ & 3 & 45 & 1.69 & 0.18 \\
$\quad$ Time of day & 4 \\
Rumination & & 45 & 0.32 & 0.57 \\
Age & 1 & 45 & 0.60 & 0.44 \\
Group & 1 & 45 & 2.73 & 0.06 \\
Time of day & 3 & &
\end{tabular}

${ }^{1}$ CowManager SensOor ear tag (Agis, Harmelen, the Netherlands).

${ }^{2}$ Age was calculated as the time in months from each heifer's birthdate.

${ }^{3}$ Group 1 heifer calves $(\mathrm{n}=24)$ were housed on straw-bedded pack and fed alfalfa hay and calf starter. Group 2 heifers $(\mathrm{n}=25)$ were fed a TMR, confirmed pregnant, and housed in freestalls bedded with composted mulch.

${ }^{4}$ Observation times were 1500, 1700, 1900, and $2100 \mathrm{~h}$. 
One potential shortcoming of this study was the absence of a test to determine potential variability contributed by the observer. One trained observer was used in this study, to decrease potential interobserver variability. This method was chosen to mitigate variability but might also have contributed to differences observed in this study. The observer in the current study was trained by experienced researchers and followed the explanations of Schirmann et al. (2009), Bikker et al. (2014), and Borchers et al. (2016) as closely as possible. We feel these methods were sufficient to effectively capture the observations used for comparison in this study. Additionally, future studies will need to account for differences in technology versions. CowManager version 7.0 was used in this study, but any future hardware or software updates could positively or negatively affect technology performance.

To our knowledge, this is the first evaluation of the CowManager technology using dairy heifers. Based on the CCC for rumination and feeding behavior, the system underperformed previously completed studies in mature dairy cattle but still had a favorable CCC for feeding time when compared with visual observations. The CowManager SensOor was more effective at recording feeding behavior than rumination behavior in dairy heifers and therefore can be used to provide relatively accurate measures of feeding time in heifers, but its rumination time function should be used with caution.

\section{ACKNOWLEDGMENTS}

The authors thank CowManager for allowing us to use their technology in the study and for their assistance with the CowManager SensOor system. We also thank the dairy staff at the Purina Animal Nutrition Center, especially Chris Joslin, for their help and cooperation. This project was the result of a cooperative research project with the Purina Animal Nutrition Center and the University of Kentucky.

\section{REFERENCES}

Bikker, J. P., H. van Laar, P. Rump, J. Doorenbos, K. van Meurs, G. M. Griffioen, and J. Dijkstra. 2014. Technical note: Evaluation of an ear-attached movement sensor to record cow feeding behavior and activity. J. Dairy Sci. 97:2974-2979.

Borchers, M. R., Y. M. Chang, I. C. Tsai, B. A. Wadsworth, and J. M. Bewley. 2016. A validation of technologies monitoring dairy cow feeding, rumination, and lying behaviors. J. Dairy Sci. 99:7458 7466 .

Bristow, D. J., and D. S. Holmes. 2007. Cortisol levels and anxietyrelated behaviors in cattle. Physiol. Behav. 90:626-628.

DeVries, T. J., K. A. Beauchemin, F. Dohme, and K. S. SchwartzkopfGenswein. 2009. Repeated ruminal acidosis challenges in lactating dairy cows at high and low risk for developing acidosis: Feeding, ruminating, and lying behavior. J. Dairy Sci. 92:5067-5078.

Duffield, T. 2000. Subclinical ketosis in lactating dairy cattle. Vet. Clin. North Am. Food Anim. Pract. 16:231-253.

Edwards, J. L., and P. R. Tozer. 2004. Using activity and milk yield as predictors of fresh cow disorders. J. Dairy Sci. 87:524-531.

Fogsgaard, K. K., C. M. Rentved, P. Sørensen, and M. S. Herskin. 2012. Sickness behavior in dairy cows during Escherichia coli mastitis. J. Dairy Sci. 95:630-638.

Friedman, H. 1982. Simplified determinations of statistical power, magnitude of effect and research sample sizes. Educ. Psychol. Meas. 42:521-526.

Goldhawk, C., N. Chapinal, D. M. Veira, D. M. Weary, and M. A. G. von Keyserlingk. 2009. Prepartum feeding behavior is an early indicator of subclinical ketosis. J. Dairy Sci. 92:4971-4977.

Hansen, S. S., P. Norgaard, C. Pedersen, R. J. Jorgensen, L. S. B. Mellau, and J. D. Enemark. 2003. The effect of subclinical hypocalcaemia induced by $\mathrm{Na}_{2}$ EDTA on the feed intake and chewing activity of dairy cows. Vet. Res. Commun. 27:193-205.

Lin, L. I. 1989. A concordance correlation coefficient to evaluate reproducibility. Biometrics 45:255-268.

Owens, F. N., D. S. Secrist, W. J. Hill, and D. R. Gill. 1998. Acidosis in cattle: A review. J. Anim. Sci. 76:275-286.

Schirmann, K., N. Chapinal, D. M. Weary, W. Heuwieser, and M. A. G. von Keyserlingk. 2011. Short-term effects of regrouping on behavior of prepartum dairy cows. J. Dairy Sci. 94:2312-2319.

Schirmann, K., M. A. von Keyserlingk, D. M. Weary, D. M. Veira, and W. Heuwieser. 2009. Technical note: Validation of a system for monitoring rumination in dairy cows. J. Dairy Sci. 92:6052-6055.

Schirmann, K., D. M. Weary, W. Heuwieser, N. Chapinal, R. L. A Cerri, and M. A. G. von Keyserlingk. 2016. Short communication: Rumination and feeding behaviors differ between healthy and sick dairy cows during the transition period. J. Dairy Sci. 99:9917-9924

Van Winden, S. C. L., R. Jorritsma, K. E. Müller, and J. P. T. M. Noordhuizen. 2003. Feed intake, milk yield, and metabolic parameters prior to left displaced abomasum in dairy cows. J. Dairy Sci. $86: 1465-1471$. 